\title{
Embarazo y tratamiento con metadona
}

\author{
Françoise Facy*, Myriam Rabaud**, Marion Andry*** \\ * Director de Investigación Inserm (Institut National de la Santé et de la Recherche Médicale) \\ * Ingeniero de Investigación Inserm (Institut National de la Santé et de la Recherche Médicale) \\ *** Documentalista de Investigación Inserm (Institut National de la Santé et de la Recherche Médicale) \\ Enviar correspondencia: \\ Françoise Facy XR302 - 44, chemin de Ronde 78116 Le Vésinet Cedex. France
}

\section{RESUMEN}

Se analizan las necesidades específicas de las mujeres toxicómanas embarazadas al principio y durante el tratamiento con metadona partiendo de una muestra de 119 mujeres que solicitan tratamiento, dentro de un estudio nacional hecho en Francia sobre el conjunto de toxicómanos en tratamiento. El $66 \%$ de la muestra son adictas a la heroína y el $15 \%$ a la buprenorfina. Durante el tratamiento se pudo constatar un aumento de las dosis medias prescritas de metadona, lo cual ha favorecido una mejora de la situación general y una disminución de las consultas necesarias.

Los estudios internacionales tanto con mujeres cocainómanas como con heroinómanas indican, como en nuestro estudio, como tanto la demanda de tratamiento como el seguimiento del tratamiento con metadona viene facilitado si el equipo de tratamiento aporta apoyo psicoeducativo, en conexión con el ambiente familiar cuando sea posible, para facilitar que exista un proyecto para el futuro niño.

Nuestro estudio muestra como las mujeres embarazadas piden ayuda a los centros especializados, pero que en ocasiones las dificultades que surgen al poner en marcha el tratamiento llevan a abandonos precoces.

El embarazo debe considerarse un momento privilegiado para abordar los problemas de abuso de drogas y un momento en que es más fácil motivar para la inclusión en tratamiento. Debería mejorar la articulación entre los equipos de toxicomanías y los de ginecología y pediatría.

Palabras clave: toxicomanía, mujeres embarazo, Metadona, epidemiología.

\section{ABSTRACT}

Analysis of the specific needs of pregnant drug-dependent women at the commencement of and during treatment with methadone, on the basis of a sample of 119 women who requested treatment, in a national study in France on drug addicts undergoing treatment. $66 \%$ of the sample were addicted to heroin and $15 \%$ to buprenorphine. During treatment, it could be seen that there was an increase in the average prescribed doses of methadone, which favoured an improvement in the general situation and a reduction in the necessary consultations.

In line with our study, international studies with both women cocaine dependents and heroin dependents also indicate how both the demand for treatment and the continuance of the treatment with methadone was easier if the treatment team provided psychoeducational support, in connection with the family environment, wherever possible, to facilitate the existence of a project for the future child.

Our study shows that pregnant women do ask for assistance from specialist centres but, on occasion, the difficulties that arise when undergoing treatment leads them to abandon it early.

Pregnancy should be considered to be a privileged moment to approach the problems of drug abuse and as a moment during which it is easier to motivate women to take treatment. The articulation between the drug dependency, gynaecological and paediatric teams must be improved.

Key words: drug dependency, women, pregnancy, methadone, epidemiology.

\section{RÉSUMÉ}

Les besoins spécifiques des femmes toxicomanes en période de grossesse sont analysés au début et au cours du traitement avec la méthadone sur un échantillon de 119 femmes enceintes demandeuses de traitement, à partir de l'étude nationale française de l'ensemble des toxicomanes traités. Pour $66 \%$ d'entre elles le produit principal de dépendance est l'héroïne et pour le 15\% la buprénorphine. Durant la grossesse on constate une augmentation moyenne des doses de méthadone prescrites, cela abouti à une évolution générale favorable et à une diminution des consultations.

Les études internationales concernant des femmes cocaïnomanes surtout, mais aussi les observations cliniques sur les héroïnomanes indiquent, comme dans notre étude, une demande de soins facilitée et un meilleur suivi du traitement avec la méthadone, si l'équipe apporte écoute et soutien psycho-éducatifs en lien avec l'entourage familial si possible, pour positiver le projet d'enfant.

Notre étude montre que les femmes enceintes sont demandeuses de soins auprès des centres spécialisés en toxicomanie, mais les difficultés rencontrées dans la mise en place et le suivi du traitement entraînent souvent des sorties précoces.

La grossesse reste un moment privilégié de repérage des abus de drogues et un temps de motivation important pour le "traitement»; elle devrait permettre une meilleure articulation entre les équipes spécialisées en toxicomanie et en néonatalogie.

Mots clés: Toxicomanie, Femmes, Grossesse, Méthadone, Epidémiologie. 


\section{INTRODUCTION}

$\mathbf{L}$ a France a peu développé la prescription de produit de substitution aux toxicomanes jusque dans les années 80. La méthadone est restée longtemps limitée à quelques centres expérimentaux. La fin des années 80 a connu des bouleversements importants avec la diffusion des infections virales, l'élargissement de la prescription à tous les centres spécialisés des traitements s'est faite progressivement au début des années 90.

La politique de substitution depuis 1995 s'appuie sur deux médicaments bénéficiant d'une autorisation de mise sur le marché spécifique pour le traitement des pharmaco-dépendances aux opiacés: la méthadone et le Subutex ${ }^{\circledR}$. Les protocoles de prise en charge sont différents et tiennent surtout compte des spécificités des lieux de soins, spécialisés ou non, pour des patients toxicomanes. Ces traitements constituent une modalité de prise en charge et s'intègrent dans une stratégie thérapeutique d'ensemble de la dépendance.

Les objectifs de cette prescription sont de favoriser: une insertion dans un processus thérapeutique et une amélioration du suivi médical d'éventuelles pathologies associées à la toxicomanie d'ordre psychiatrique et/ou somatique; une stabilisation de la consommation de drogues issues du marché illicite (notamment l'héroïne) et la diminution du recours à la voie injectable, source de transmission virale et infectieuse; une insertion sociale. L'objectif ultime est de permettre à chaque patient d'élaborer une vie sans dépendance.

Après une période d'augmentation progressive des autorisations de prescription, tous les centres spécialisés de soins aux toxicomanes depuis janvier 1995 peuvent prescrire et délivrer de la méthadone. Suite à l'autorisation de mise sur le marché de ce médicament, un dispositif rigoureux permet d'associer les médecins généralistes à ce traitement après une première phase de suivi en centre spécialisé. Ce dispositif impose que tous les centres de soins disposent du personnel nécessaire (médecins, infirmiers) pour la réalisation de cette nouvelle modalité de prise en charge.

La première phase du traitement s'exerce en centre spécialisé de soins aux toxicomanes où interviennent des équipes pluridisciplinaires. Des dosages urinaires permettent la vérification de l'intoxication aux opiacés. Le traitement une fois stabilisé, peut être pris en relais par un médecin généraliste. Pour les femmes enceintes, les recommandations en matière de traitement de substitution portent principalement sur la méthadone, plutôt que sur le Subutex ${ }^{\circledR}$, dont I'usage est plus récent (Wieviorka, 1994).

Les femmes représentent un groupe minoritaire dans le champ de la toxicomanie, comme de I'alcoolisme. Leurs difficultés d'accès aux soins, du fait même d'une certaine marginalisation par rapport aux hommes, apparaissent renforcées; la grossesse et la maternité sont des facteurs qui tendent à augmenter leurs difficultés de recours à des soins spécialisés en toxicomanie. Des comparaisons entre femmes dépistées en prison et femmes suivies en centre de soins montrent l'augmentation de difficultés pour les mères toxicomanes, et leur manque de suivi par des équipes spécialisées.

\section{OBJECTIFS ET MÉTHODOLOGIE DE L’ÉTUDE}

L'objectif d'une prescription de traitement à la méthadone des femmes enceintes toxicomanes est de faciliter une prise en charge plus globale, permettant à terme un développement de la relation mère-enfant satisfaisant.

L'étude nationale française sur l'ensemble des toxicomanes mis sous traitement à la méthadone depuis 1995 a permis I'obtention de nombreuses données épidémiologiques. Des sous-groupes ont pu y être analysés permettant d'une part de situer la place des femmes toxicomanes dans les centres spécialisés, de les comparer à des femmes dépistées en prison et d'examiner les particularités des mères toxicomanes. L'étude des femmes enceintes bénéficiant de traitement avec la méthadone montre l'évolution de leur santé et leur adaptation sociale permettant d'évaluer l'impact d'un suivi dans un contexte de centre spécialisé. La confrontation de ces données devrait permettre des recommandations en matière de dépistage, traitement et suivi des femmes toxicomanes, au cours de la grossesse en particulier.

\section{RÉSULTATS}

\section{PLACE DES FEMMES TOXICOMANES DANS LES STRUCTURES DE SOINS}

\subsection{Comparaison des femmes vues dans les centres de soins $(n=1764)$ et en prison ( $n=183)$}

\section{Données socio-démographiques}

En prison, les femmes toxicomanes sont bien moins nombreuses que dans les centres de soins, elles sont cependant deux fois plus nombreuses que la population féminine incarcérée non toxicomane. Les étrangères représentent $12 \%$ de la population et sont bien plus nombreuses que dans les centres de soins ( $5 \%$ en moyenne). $52 \%$ des femmes toxicomanes vues en prison ont moins de 25 ans, et $21 \%$ plus de 30 ans; dans les centres de soins, $37 \%$ seulement ont moins de 25 ans, et l'âge moyen est de 27 ans. 
Le logement est autofinancé pour $43 \%$ des femmes vues en prison, les sujets sans domicile fixe sont peu nombreux (10\%), comme dans la clientèle des centres (7\%). La situation matrimoniale des femmes toxicomanes incarcérées montre que presque la moitié sont célibataires, $42 \%$ vivent en couple: la notion de couple constitué plus tôt caractérise un peu plus souvent les toxicomanes incarcérées. Par contre, $44 \%$ des femmes toxicomanes incarcérées sont parents, plus que dans les centres de soins (35\%).

L'entourage familial des femmes incarcérées est marqué par des risques accrus de toxicomanie: 29\% ont un membre de leur fratrie toxicomane et $55 \%$ ont un partenaire toxicomane.

Les risques liés à la santé du sujet et de son environnement proche sont ainsi soulignés et renforcent les préoccupations de Santé Publique vis-àvis de la transmission du VIH et des maladies infectieuses (hépatite, tuberculose,...).

L'activité professionnelle est rarement continue $(17 \%)$, le manque de qualification professionnelle touche $49 \%$ des femmes en prison.

Le niveau d'études apparaît plus élevé chez les sujets demandeurs de soins: $18 \%$ ont un niveau Bac ou plus parmi la "clientèle " des centres spécialisés. Pour elles, l'activité professionnelle est le plus souvent intermittente (37\%).

Ainsi, les deux groupes de femmes toxicomanes, vues dans les centres de soins ou vues en prison sont très différents sur les caractéristiques sociodémographiques principales: âge, niveau d'études, nationalité, emploi. Un certain nombre d'effets de sélection sont caractéristiques de l'incarcération.

\section{Situation juridique}

Parmi les femmes toxicomanes incarcérées, 64\% sont prévenues et $32 \%$ sont condamnées. $55 \%$ ont commis un délit en rapport avec I'ILS (infraction à la législation sur les stupéfiants). Le délit est en relation exclusive avec la drogue pour $52 \%$ des cas, en relation directe avec la drogue pour $24 \%$ des cas, et sans relation pour $11 \% .47 \%$ sont récidivistes. $43 \%$ ont été incarcérées pour la première fois avant 20 ans. L'importance des comportements de délinquance et des difficultés précoces avec la justice est ainsi soulignée, survenant bien avant les comportements de toxicomanie pour un nombre non négligeable de sujets. $39 \%$ ont déjà eu une injonction thérapeutique.

Dans les centres spécialisés, 20\% des femmes toxicomanes ont été incarcérées, les hommes ayant déjà été incarcérés sont deux fois plus nombreux en proportion.

\section{Données médicales}

Seules 5\% des femmes toxicomanes vues en prison disent ne pas avoir effectué un test VIH; $12 \%$ sont séropositives.
Au niveau des centres spécialisés, 56\% ont effectué un test. On estime pour les consultants une prévalence de la séropositivité moyenne de $27 \%$.

$21 \%$ (18\% dans les centres spécialisés) des femmes toxicomanes incarcérées ont eu des problèmes d'overdose, 24\% d'hépatite virale (B ou C). Les problèmes de santé mentale se révèlent fréquents chez les femmes toxicomanes incarcérées: $37 \%$ on fait une tentative de suicide ( $28 \%$ dans les centres de soins, dont 1 sur 5 avant la toxicomanie). Plus d'une femme sur trois a eu une consultation psychiatrique (dont 1 sur 5 avant la toxicomanie).

Ces chiffres semblent être minimaux, l'imprécision des réponses reflète un intérêt variable des équipes et une connaissance hétérogène de l'état de santé des patientes.

\section{Données toxicologiques}

La plupart des femmes toxicomanes associent différents produits. Pour les héroïnomanes consultants en centres spécialisés, la durée de dépendance est en moyenne de 7 ans, la durée totale d'intoxication est en moyenne de 10 ans. Ces durées moyennes sont légèrement inférieures pour les héroïnomanes incarcérées, la prison intervenant plus tôt dans leur rencontre avec les structures. Chez les femmes incarcérées, versus traitées, les produits pris en association sont I'héroïne (90\% vs 68\%), le cannabis (34\% vs 38\%), les médicaments (30\% vs 32\%), la cocaïne (32\% vs $11 \%)$, I'alcool (10\% vs $26 \%$ ) et la codéine (12\% vs 25\%).

Le produit de début le plus souvent cité est d'abord le cannabis (39\% vs 36\%), puis I'héroïne (26\% vs $26 \%)$, I'alcool (6\% vs $7 \%$ ).

Les profils d'intoxication des femmes rencontrées en prison ou en centres de soins sont très proches dans leur déroulement chronologique entre première intoxication et dépendance. L'héroïne est le produit dominant, le cannabis vient ensuite.

Pour les produits de début, le cannabis reste le plus fréquent, mais un nombre important de femmes toxicomanes disent avoir commencé par I'héroïne.

\subsection{Comparaison des mères toxicomanes vues dans les centres de soins $(n=613)$ et en prison $(\mathrm{n}=\mathbf{8 0})$}

Bien plus souvent que les hommes toxicomanes, les femmes toxicomanes ont des enfants; c'est parmi les femmes incarcérées, que les mères toxicomanes sont relativement plus nombreuses que parmi les toxicomanes reçues en milieu sanitaire, spécialisé ou non.

Quand elles ont des enfants, les femmes toxicomanes incarcérées ont aussi un nombre d'enfants un peu plus élevé (34\% en ont deux ou plus), 
que les femmes traitées en centres de soins (30\%). Ceci est à rapprocher du fait qu'en prison les mères toxicomanes sont en moyenne plus jeunes: $44 \%$ ont moins de 25 ans contre $21 \%$.

\section{Données socio-démographiques}

La nationalité des mères toxicomanes incarcérées est étrangère pour 15\% (vs 5\%). Elles vivent en couple à $53 \%$ (vs 39\%). Elles ont un logement indépendant de leur famille pour $51 \%$, comme dans les centres, et sont sans domicile fixe à $10 \%$ (vs $8 \%$ ). Seules $17 \%$ ont un travail continu (vs 12\%). La moitié n'ont aucune qualification professionnelle (vs 28\%). Pour les mères toxicomanes incarcérées, le partenaire est toxicomane dans 58\% des cas et quand elles ont une fratrie, la présence de toxicomanes est signalée pour 39\% des cas.

\section{Données médicales}

Le test VIH n'est pas effectué pour $3 \%$ des mères toxicomanes incarcérées (vs 18\%), le résultat n'est pas précisé pour $21 \%$ (vs 23\%), il est positif pour $8 \%$ (vs $29 \%)$. Une overdose est notée par $18 \%$ des mères incarcérées, $6 \%$ déclarent une maladie sexuellement transmissible. 24\% signalent une hépatite (B ou C) (vs $22 \%$ ) et $10 \%$ d'autres affections.

Sur le plan de la santé mentale, $40 \%$ ont effectué une tentative de suicide (vs 30\%), 38\% ont eu une consultation psychiatrique (dont 1 sur 6 avant toxicomanie). $30 \%$ ont eu une hospitalisation en psychiatrie.

\section{Situation juridique}

Les deux tiers des mères toxicomanes incarcérées sont prévenues. Le délit concerne une infraction à la législation sur les stupéfiants pour la moitié d'entre elles. Le délit est sans relation avec la drogue pour $14 \%$, montrant ainsi le rôle de dépistage des toxicomanes rempli par les antennes en prison.

La moitié des mères sont récidivistes, l'âge moyen à la première incarcération étant de 19 ans. 48\% ont déjà une injonction thérapeutique.

\section{Données toxicologiques}

Les produits utilisés par les mères toxicomanes incarcérées sont essentiellement I'héroïne (91\% vs $72 \%$ ), le cannabis (36\% vs 34\%), la cocaïne (33\% vs $10 \%$ ) et les médicaments (31\% vs 32\%).

Le produit de début est le cannabis (41\% vs 33\%) puis l'héroïne (25\% vs 29\%). Les durées d'intoxication dépassent 5 ans pour quatre mères toxicomanes sur cinq.

Le suivi dans un centre de soins concerne moins d'une mère toxicomane incarcérée sur quatre, montrant ainsi le faible recours aux structures de cette catégorie de population.

Par contre dans les centres de soins, plus de la moitié des mères toxicomanes ont déjà eu des contacts antérieurs avec des centres, $40 \%$ ont déjà effectué un sevrage, mais ceci témoigne également de rechutes nombreuses.

\section{EVOLUTION DES FEMMES ENCEINTES TOXICO- MANES TRAITEES A LA METHADONE}

Dans l'étude nationale menée depuis 1995 auprès des centres prescripteurs de méthadone, les femmes toxicomanes enceintes qui demandent un traitement, constituent un sous-échantillon de 119 sujets. Leurs dossiers de bilans de santé et de suivi du traitement sont analysés globalement sur une période inférieure à un an. La durée en mois de traitement en méthadone est de 1 mois pour 29\% de l'échantillon, de 1 à 5 mois pour $37 \%$ et de 6 à 11 mois pour 34\% des femmes enceintes étudiées.

\section{Situation initiale}

Au niveau toxicologique et médical (tableaux 1 et 2 respectivement)

Le produit principal de dépendance est I'héroïne pour 66\% des consultantes; pour les autres patientes, $15 \%$ indiquent le Subutex ${ }^{\circledR}$, 3\% la méthadone, 5\% la codéine. L'injection intraveineuse est (ou a été) pratiquée par $70 \%$ des sujets. $21 \%$ associent de la cocaïne, $4 \%$ de la codéine, $12 \%$ des benzodiazépines, $4 \%$ du Rohypnol ${ }^{\circledR}$ et $6 \%$ de l'alcool. Le produit de début est le cannabis (33\%), I'héroïne (45\%).

L'âge de début d'intoxication est de 17 ans en moyenne. $54 \%$ des sujets ont eu des sevrages institutionnels antérieurs à leur entrée dans le programme. Le nombre moyen de sevrages est 3. Au niveau des difficultés rencontrées, 32\% ont eu un surdosage, $40 \%$ ont fait une tentative de suicide, $12 \%$ ont eu un traumatisme physique, $22 \%$ ont eu une hospitalisation psychiatrique et $40 \%$ ont eu recours au service des urgences.

Le test de sérodiagnostic VIH est effectué dans 94\% des cas. Il est alors positif pour 7\% des patientes, parmi elles un quart ont une forme clinique majeure et seules 38\% suivent des traitements spécifiques.

Au niveau des hépatites, $21 \%$ sont touchées par I'hépatite B et $52 \%$ par l'hépatite C. $10 \%$ ont d'autres infections. Les taux de non-réponses à ces questions sont relativement élevés, montrant les difficultés à établir les situations sanitaires lors du bilan initial.

Les antécédents sont fréquents: médicaux 19\%, chirurgicaux 32\%; 9\% des sujets ont une maladie chronique et 56\% mentionnent des antécédents obstétricaux. Les traitements signalés sont des antidépresseurs pour $6 \%$, des neuroleptiques pour $4 \%$ et un autre psychotrope pour $30 \%$ des patientes. 
Tableau 1: Intoxication des femmes enceintes avant traitement

\begin{tabular}{|lcc|}
\hline $\mathbf{N}=\mathbf{1 1 9}$ & & Bilan initial \\
\hline Dernier produit principalement utilisé & héroïne & $66 \%$ \\
& Subutex & $15 \%$ \\
& codéine & $5 \%$ \\
& cocaïne & $3 \%$ \\
Age de début du produit principal & Temgésic & $<1 \%$ \\
\hline Produit secondaire 1 & méthadone & $3 \%$ \\
& moyenne [ampl.] & 21 ans [12-37] \\
& cannabis & $26 \%$ \\
& cocaïne & $21 \%$ \\
& Benzodiazépine & $12 \%$ \\
& héroïne & $12 \%$ \\
\hline Injection intra-veineuse & codéine & $4 \%$ \\
& alcool & $6 \%$ \\
\hline Produit de début & Rohypnol & $4 \%$ \\
\hline Age du produit de début & oui-actuellement & $39 \%$ \\
\hline Sevrages institutionnels antérieurs & oui-antérieurement & $31 \%$ \\
\hline Nombre de sevrages institutionnels & héroïne & $45 \%$ \\
\hline Les \% sont exprimés par rapport aux répondants. & cannabis & $33 \%$ \\
\hline
\end{tabular}

Tableau 2: Caractéristiques médicales des femmes enceintes avant et au cours du traitement

\begin{tabular}{|lccc|}
$\mathbf{N}=119$ & Bilan initial & $\begin{array}{c}\text { Etat actuel à } \\
<12 \text { mois de méthadone }\end{array}$ \\
\hline Surdosage & oui & $32 \%$ & $<1 \%$ (depuis l'entrée) \\
Tentative de suicide & oui & $40 \%$ & $2 \%$ (depuis l'entrée) \\
Traumatisme physique & oui & $12 \%$ & $<1 \%$ (depuis l'entrée) \\
Hospitalisation en psychiatrie & oui & $22 \%$ & $6 \%$ (depuis l'entrée) \\
Recours au service d'urgence & oui & $40 \%$ & $6 \%$ (depuis l'entrée) \\
\hline Test de sérodiagnostic VIH effectué & oui & $94 \%$ & $96 \%$ \\
Si test effectué: résultat & positif & $7 \%$ & $7 \%$ \\
& attente & $<1 \%$ & $<1 \%$ \\
Si test positif, infection à VIH & forme asymptom. & $50 \%$ & $63 \%$ \\
& clinique min. & $13 \%$ & $13 \%$ \\
Si test positif, traitements spécifiques & clinique maj. & $25 \%$ & $50 \%$ \\
& oui & $38 \%$ & $38 \%$
\end{tabular}




\begin{tabular}{|c|c|c|c|}
\hline$N=119$ & & Bilan initial & $\begin{array}{c}\text { Etat actuel à } \\
<12 \text { mois de méthadone }\end{array}$ \\
\hline \multirow[t]{3}{*}{ Si test positif, suivi } & régulier & $63 \%$ & $75 \%$ \\
\hline & irrégulier & $25 \%$ & $13 \%$ \\
\hline & inconnu & $13 \%$ & $13 \%$ \\
\hline \multirow[t]{3}{*}{ Hépatite virale B ou C } & oui actuelle & $25 \%$ & $43 \%$ \\
\hline & oui antérieure & $7 \%$ & $5 \%$ \\
\hline & non fait & $7 \%$ & $5 \%$ \\
\hline \multirow[t]{4}{*}{ Hépatite $B$} & oui actuelle & $7 \%$ & $12 \%$ \\
\hline & oui antérieure & $14 \%$ & $14 \%$ \\
\hline & non & $68 \%$ & $66 \%$ \\
\hline & non fait & $2 \%$ & $2 \%$ \\
\hline \multirow[t]{4}{*}{ Hépatite C } & oui actuelle & $43 \%$ & $48 \%$ \\
\hline & oui antérieure & $9 \%$ & $6 \%$ \\
\hline & non & $41 \%$ & $40 \%$ \\
\hline & non fait & $2 \%$ & $2 \%$ \\
\hline Tuberculose actuelle & oui & $0 \%$ & $0 \%$ \\
\hline Autres infections & oui & $10 \%$ & $10 \%$ \\
\hline Maladie chronique & oui & $9 \%$ & $10 \%$ \\
\hline Antécédents médicaux & oui & $19 \%$ & $21 \%$ \\
\hline Antécédents chirurgicaux & oui & $32 \%$ & $32 \%$ \\
\hline Antécédents obstétricaux & oui & $56 \%$ & $84 \%$ \\
\hline Traitement psychotrope antidépresseur & oui & $6 \%$ & $9 \%$ \\
\hline Traitement psychotrope neuroleptique & oui & $4 \%$ & $5 \%$ \\
\hline Traitement psychotrope autre & oui & $30 \%$ & $35 \%$ \\
\hline
\end{tabular}

Au niveau socio-démographique (tableaux 3 et 4)

Dans l'ensemble des prescriptions de méthadone depuis 1995, la proportion de femmes est de 30\%, les femmes enceintes étant $11 \%$, ce qui représente un groupe relativement important. $43 \%$ des patientes enceintes sont célibataires, $42 \%$ vivent en couple, $57 \%$ sont déjà mères. Leur entourage habituel comprend un conjoint (50\%), des enfants (32\%), des parents (37\%), $8 \%$ vivent en institution.

Le niveau scolaire est pour $49 \%$ inférieur au secondaire (BEPC ou CAP) et $17 \%$ ont un niveau bac ou plus. $21 \%$ bénéficient du RMI (Revenu Minimum d'Insertion), seulement $8 \%$ n'ont aucune protection sociale. Pour l'activité professionnelle, $7 \%$ en ont une continue, 38\% intermittente. Les actives sont à $64 \%$ des employées.

L'origine des ressources indique pour 7\% un emploi, pour $53 \%$ une aide; $13 \%$ n'ont aucune ressource et $27 \%$ mentionnent une source de revenus autre que le travail ou des aides. $20 \%$ ont un endettement non maîtrisé. $42 \%$ des patientes ont au moins une personne à charge (souvent un enfant).

Au niveau légal, les difficultés sont nombreuses, puisque $29 \%$ ont été incarcérées. La médiane de la durée d'incarcération est de 10 mois et la moyenne 18 mois.

Le cumul des difficultés est également souligné pour les femmes toxicomanes enceintes dans d'autres pays, aux Etats-Unis sur le plan socio-économique, qui recommande un repérage des situations pour prévenir les risques de maltraitance des enfants.

\section{Au niveau de la substitution (tableau 5)}

Parmi les femmes enceintes débutant le traitement, $38 \%$ ont déjà été inclues dans un programme agréé de substitution: $27 \%$ ont reçu de la méthadone et $46 \%$ ont déjà pris de la buprénorphine.

De nombreuses prises en charge antérieures sont observées, de natures diverses (sevrage, substitution 
Tableau 3: Données socio-démographiques des femmes

enceintes au début et au cours du traitement

\begin{tabular}{|c|c|c|c|}
\hline$N=119$ & & Bilan initial & $\begin{array}{c}\text { Etat actuel à } \\
<12 \text { mois de méthadone }\end{array}$ \\
\hline \multirow[t]{3}{*}{ Nationalité } & française & $93 \%$ & \\
\hline & étrangère & $6 \%$ & \\
\hline & non précisée & $<1 \%$ & \\
\hline \multirow[t]{5}{*}{ Situation familiale } & célibataire & $43 \%$ & \\
\hline & union libre & $37 \%$ & \\
\hline & mariée & $5 \%$ & \\
\hline & veuve & $3 \%$ & \\
\hline & séparée & $10 \%$ & \\
\hline \multirow[t]{8}{*}{ Niveau scolaire atteint } & primaire & $8 \%$ & \\
\hline & second/BEPC & $24 \%$ & \\
\hline & technique/CAP & $17 \%$ & \\
\hline & second/2ème cycle & $11 \%$ & \\
\hline & technique/BEP & $23 \%$ & \\
\hline & Bac général & $12 \%$ & \\
\hline & Bac technique & $<1 \%$ & \\
\hline & $\mathrm{Bac}+3 \mathrm{ou}>$ & $4 \%$ & \\
\hline Age actuel & moyenne [ampl.] & 32 ans [19-44] & \\
\hline \multirow[t]{4}{*}{ Nombre d'enfants } & 2 et + & $21 \%$ & \\
\hline & 1 & $36 \%$ & \\
\hline & aucun & $30 \%$ & \\
\hline & non réponse & $13 \%$ & \\
\hline \multirow[t]{4}{*}{ Protection sociale actuelle } & RMI & $21 \%$ & $19 \%$ \\
\hline & aucune & $8 \%$ & $6 \%$ \\
\hline & prot. personnelle & $4 \%$ & $4 \%$ \\
\hline & prot. autre & $67 \%$ & $71 \%$ \\
\hline Qualification professionnelle & oui & $47 \%$ & $48 \%$ \\
\hline \multirow[t]{4}{*}{ Activité professionnelle } & continue & $7 \%$ & $7 \%$ \\
\hline & aucune/jamais & $25 \%$ & $26 \%$ \\
\hline & intermittente & $38 \%$ & $37 \%$ \\
\hline & interruption & $16 \%$ & $16 \%$ \\
\hline \multirow[t]{6}{*}{ Catégorie socio-professionnelle } & employés & $20 \%$ & $20 \%$ \\
\hline & employés admin. & $15 \%$ & $15 \%$ \\
\hline & employ. commerce & $29 \%$ & $29 \%$ \\
\hline & santé/social & $6 \%$ & $5 \%$ \\
\hline & chômeur & $2 \%$ & $2 \%$ \\
\hline & prostituée & $0 \%$ & $0 \%$ \\
\hline
\end{tabular}


Tableau 4: Aspects sociaux des femmes enceintes au début et au cours du traitement

\begin{tabular}{|c|c|c|c|}
\hline$N=119$ & & Bilan initial & $\begin{array}{c}\text { Etat actuel à } \\
<12 \text { mois de méthadone }\end{array}$ \\
\hline \multirow[t]{6}{*}{ Logement } & auto-financé & $50 \%$ & $63 \%$ \\
\hline & chez parents & $6 \%$ & $3 \%$ \\
\hline & chez un tiers & $12 \%$ & $9 \%$ \\
\hline & indépendant & $4 \%$ & $5 \%$ \\
\hline & $S D F$ & $6 \%$ & $3 \%$ \\
\hline & dans un foyer & $11 \%$ & $8 \%$ \\
\hline \multirow[t]{2}{*}{ Entourage habituel } & oui & $50 \%$ & $45 \%$ \\
\hline & oui & $32 \%$ & $40 \%$ \\
\hline \multirow[t]{3}{*}{ Nbre de personnes dépendantes du sujet } & 0 & $58 \%$ & \\
\hline & 1 & $31 \%$ & \\
\hline & 2 et + & $11 \%$ & \\
\hline \multirow[t]{4}{*}{ Origine des ressources actuelles } & aide seule & $53 \%$ & $52 \%$ \\
\hline & sans ressource & $13 \%$ & $10 \%$ \\
\hline & emploi seul & $7 \%$ & $8 \%$ \\
\hline & autre & $27 \%$ & $30 \%$ \\
\hline \multirow[t]{3}{*}{ Endettement } & maîtrisé & $19 \%$ & $20 \%$ \\
\hline & non maîtrisé & $20 \%$ & $20 \%$ \\
\hline & aucun & $62 \%$ & $61 \%$ \\
\hline Incarcération & oui & $29 \%$ & - \\
\hline \multirow[t]{2}{*}{ Durée totale d'incarcération $(n=35)$} & moyenne & 18 mois & - \\
\hline & médiane & 10 mois & \\
\hline Gravité des conduites de délinquance & moyenne [ampl.] & $2[1-7]$ & $2[0-7]$ \\
\hline
\end{tabular}

en ambulatoire, inclusion dans un protocole méthadone) dans des parcours où les rechutes sont nombreuses.

Parmi les 34 femmes enceintes qui disent avoir pris un produit à visée substitutive, $14 \%$ indiquent la codéine, $46 \%$ le temgésic (ou subutex), $7 \%$ d'autres opiacés.

Au bout du premier mois de prescription de méthadone, la dose moyenne est $50 \mathrm{mg}$ (médiane 45) et il y a 5 venues hebdomadaires en moyenne dans le centre.

A l'entrée, le bilan urinaire est positif à la méthadone (20\%), à un autre opiacé $(69 \%)$, à un autre toxique (39\%).

Au niveau de la gravité des comportements (tableau 6)
Différentes échelles visuelles sont utilisées, cotées de 1 à 7 , pour mesurer les attitudes de prévention (risques sanguin et sexuel), les troubles anxieux et psychologiques, les comportements de délinquance et I'insatisfaction des relations familiales et sociales.

Pour les attitudes de prévention, les risques de contamination sanguine et sexuelle sont en moyenne de 2 et 3.

Pour les données psychologiques:

- troubles anxieux: moyenne 3

- troubles dépressifs: moyenne 3

- troubles psychotiques: moyenne 1

- troubles du comportement: moyenne 2

Les niveaux d'insatisfactions familiale et sociale indiquent une moyenne respectivement de 4 et de 3 . Pour les conduites de délinquance, la gravité est en moyenne de 2 . 
Tableau 5: Suivi du traitement avec substitution chez les femmes enceintes au début et au cours du traitement

$\mathrm{N}=119$

Bilan initial

Etat actuel à

$<12$ mois de méthadone

\section{A la fin du ${ }^{\text {er }}$ mois}

Prise de méthadone

dans les 3 derniers mois

$20 \%$

antérieurement

non

$7 \%$

$73 \%$

Prise de buprénorphine

dans les 3 derniers mois

$27 \%$

antérieurement

$19 \%$

non

$54 \%$

$23 \%$

Prise d'un autre produit de substitution

dans les 3 derniers mois antérieurement

$8 \%$

$69 \%$

Inclus dans un protocole de substitution

non

$38 \%(n=40)$

Produit à visée de substitution (prise)

oui

$34 \%(n=34)$

Produit à visée de substitution utilisé

Dose de méthadone (mg)

oui

Subutex

$46 \%$

codéine

$14 \%$

temgésic

$7 \%$

méthadone

$14 \%$

autres opiacés

$7 \%$

moyenne [ampl.]

$50[10-130]$

58 [25-100]

médiane

moyenne

45

55

Nombre de venues hebdomadaires

médiane

5

3

5

2

Bilan urinaire + , à la méthadone

oui

$20 \%$

oui $\quad 69 \%$

Bilan urinaire + , à un autre opiacé

oui

$39 \%$

Les \% sont exprimés par rapport aux répondants.

Tableau 6: Suivi des comportements chez les femmes enceintes au début et au cours du traitement

$$
N=119
$$

Bilan initial

Etat actuel à

$<12$ mois de méthadone

Risque de contamination sanguine

Risque de contamination sexuelle

Echelle troubles dépressifs

Echelle troubles anxieux

Echelle troubles du comportement

Echelle troubles psychotiques

moyenne [ampl.]

2 [0-7] (actuel)

moyenne [ampl.]

2 [1-7]

2 [0-7] (actuel)

moyenne [ampl.]

3 [1-7]

3 [1-6] (actuelle)

moyenne [ampl.]

3 [1-7]

3 [1-7] (actuelle)

moyenne [ampl.]

3 [1-7]

2 [1-6] (actuelle)

moyenne [ampl.]

2 [1-7]

1 [1-7] (actuelle)

Echelle d'insatisfactions familiales

moyenne [ampl.]

1 [1-7]

4 [1-7]

moyenne [ampl.]

4 [1-7]

$3[1-7]$

$3[1-7]$

Les \% sont exprimés par rapport aux répondants. 


\section{Analyse de suivi du traitement}

Présence dans le centre primo-prescripteur (tableau 7)

Suivant les informations disponibles à I'Inserm [Institut National de la Santé et de la Recherche Médicale] (en juin 2001), 29\% des 119 femmes étudiées sont sorties du centre au bout d'un mois, $37 \%$ sont suivies moins de six mois et $34 \%$ sont suivies de six mois à un an. $6 \%$ ont changé de centre et $2 \%$ des femmes ont été transférées vers un médecin de ville. 2 patientes sont décédées.

Caractéristiques du traitement au dernier bilan (tableaux 2, 5 et 7)

La période de suivi sous méthadone se situe entre 4 et 12 mois pour les deux tiers des patientes. Le taux de rétention apparaît relativement bon, compte tenu des nombreuses difficultés d'accès aux soins et des résistances aux consultations signalées pour les femmes enceintes (Picard, 1995).

La dose de méthadone est en moyenne de $58 \mathrm{mg}$ [médiane 55] (tableau 5). Le nombre moyen de venues hebdomadaires est 3 . La fréquence mensuelle de consultations est 2 (tableau 7).
La question des dosages est abordée par différents auteurs, la fraction quotidienne des prises, la précocité de la prescription, l'intérêt même de faibles doses.

Les prises en charge associant méthadone et suivi psychosocial sont bien plus fréquentes pour les femmes enceintes que pour l'ensemble des toxicomanes traités. L'intérêt d'interventions pluridisciplinaires est souligné dans d'autres études en Belgique, d'un " traitement amélioré " aux Etats-Unis, d'une gestion de la grossesse à partir d'expériences européennes. La nécessité de politiques adaptées est aussi soulignée. Les études plus récentes menées au Québec montrent la fragilité des mères toxicomanes et insistent sur la spécificité des interventions (Guyon et al., 2003).

Durant la grossesse, les doses de méthadone ont augmenté, parallèlement à une diminution des consultations. Les entretiens psychothérapeutiques concernent $46 \%$ des patientes. Le suivi socio-éducatif est réalisé pour $67 \%$ des sujets, bien plus souvent que pour l'ensemble des patients.

Les traitements associés sont des antidépresseurs $(9 \%)$, des neuroleptiques (5\%), d'autres psychotropes (35\%), en nette progression par rapport au début du traitement (tableau 2).

\section{Tableau 7: Suivi du traitement chez les femmes enceintes au début et au cours du traitement}

$\mathrm{N}=119$
Etat actuel à

$<12$ mois de méthadone

\section{Sortie du protocole}

Transférée vers un médecin de ville

Changement de centre

Nbre venues hebdomadaires actuelles

Fréquences mensuelles de consultations

Entretiens psychiatriques

Suivi socio-éducatif

$$
\text { oui }
$$

dont par décision médicale

$$
\text { oui }
$$

moyenne

moyenne

oui

oui
$49 \%(n=58)$

$20 \%(n=11)$

$2 \%(\mathrm{n}=2)$

$6 \%(n=7)$

3

2

$46 \%$

$67 \%$

Les \% sont exprimés par rapport aux répondants.

Ces données témoignent d'une attention accrue portée par les équipes aux femmes toxicomanes durant la grossesse.

\section{Evolution des femmes enceintes au niveau des usages de produits}

Les conduites toxicomaniaques ne sont pas systématiquement repérées lors des suivis mais les produits suivants sont signalés (tableau 8):

L'évolution des usages indique une diminution importante des opiacés. L'augmentation la plus nette concerne l'alcool, utilisé par $38 \%$ des sujets et les benzodiazépines utilisées par 50\%, comme pour l'ensemble des toxicomanes traités à la méthadone.

D'autres études confirment cette tendance à des consommations en plus de la méthadone en Australie (Fischer, 1999); la diminution d'opiacés est signalée par Boer (1994) aux Pays-Bas, Batey (1993) en Australie.

\section{Etat de santé}

Les événements survenus durant le suivi (tableau 2) sont: 
Tableau 8: Usages de produits psychoactifs chez les femmes enceintes

\begin{tabular}{|lccc|}
\hline & $\begin{array}{c}\text { En usage habituel } \\
(\mathbf{\%})\end{array}$ & $\begin{array}{c}\text { En usage intermittent } \\
(\mathbf{\%})\end{array}$ & $\begin{array}{c}\text { En usage occasionnel } \\
(\%)\end{array}$ \\
\hline Cocaïne & 7 & - & 14 \\
Alcool & 4 & 4 & 29 \\
Opiacés & 6 & 3 & 55 \\
Benzodiazépines & 30 & 10 & 10 \\
Autres produits & 9 & 4 & 26 \\
\hline
\end{tabular}

- des tentatives de suicide $2 \%$,

- des surdosages $1 \%$,

- des accidents $2 \%$,

- des traumatismes $1 \%$

- des hospitalisations psychiatriques 6\%,

- des recours aux urgences $6 \%$,

L'état infectieux connu montre que $96 \%$ ont effectué un test $\mathrm{VIH}$. 7\% sont séropositives, dont 13\% ont une forme clinique majeure. La moitié des femmes enceintes séropositives ont un traitement spécifique, plus souvent qu'au début du traitement.

Le dernier bilan montre une augmentation des hépatites, $26 \%$ ont (ou ont eu) une hépatite B et $54 \%$ une hépatite $\mathrm{C}$.

Les difficultés obstétricales sont signalées pour $84 \%$ des patientes, l'augmentation (28\%) depuis le début du traitement révèle des grossesses souvent difficiles.

Situation sociale et évolution psychologique (tableau 4)

L'origine des ressources indique une amélioration de la situation, $10 \%$ toutefois restent sans ressource. Une grande précarité de logement concerne 3\% des cas.

L'entourage familial reste important, toutefois il y a une diminution de la présence des conjoints (45\% présents) et des parents (31\% présents).

Sur les échelles de comportement et de psychopathologie, aucune différence n'est observée en moyenne, contrairement à l'ensemble des patients traités pour qui ces améliorations sont notables en moyenne, plus rapidement sur les attitudes et relations que sur l'état psychologique des patients. Toutefois, les cotations moyennes à ces échelles montrant des gravités moyennes ou faibles, le traitement à la méthadone permet de maintenir ces niveaux et de stabiliser les situations.

\section{DISCUSSION}

La question de la spécificité des femmes dans le traitement de la toxicomanie est régulièrement posée, d'autant plus qu'elles apparaissent à la fois minoritaires et concernées par les niveaux les plus élevés de toxicomanie. Les difficultés associées notamment à la période de grossesse sont actuellement mieux connues, en particulier pour la prédiction de problèmes de santé et de développement psychique des enfants.

L'évaluation des traitements est cependant commune à tous les patients au moins sur les aspects de dosage et de suivi psychosocial.

La question des dosages est abordée par différents auteurs, la fraction quotidienne des prises (Jarvis, 1999), la précocité de la prescription (premier trimestre de grossesse pour Depetrillo, 1995), l'intérêt même de faibles doses (Offidani, 1986).

Les prises en charge associant méthadone et suivi psycho-social sont bien plus fréquentes pour les femmes enceintes que pour l'ensemble des toxicomanes traités. L'intérêt d'interventions pluridisciplinaires est souligné dans d'autres études en Belgique (Picard, 1995), d'un "traitement amélioré " aux Etats-Unis (Chang, 1992), d'une gestion de la grossesse à partir d'expériences européennes (Cahiers T3E, 1997). La nécessité de politiques adaptées est soulignée par Mc Lean (1994).

Des études concordent sur la tendance à d'autres consommations en plus de la méthadone en Australie (Fischer, 1999) et ainsi qu'à la diminution d'opiacés Boer (1994) aux Pays-Bas, Batey (1993) en Australie. Leur impact sur la grossesse est plutôt décrit sur un plan clinique qu'épidémiologique (Guyon, 2003).

Le cumul des difficultés est souligné pour les femmes toxicomanes enceintes dans d'autres pays, aux Etats-Unis sur le plan socio-économique par Finnegan (1995), qui recommande un repérage des situations pour prévenir les risques de maltraitance des enfants. La reproduction de schémas de carences parentales est analysée au Québec, avec l'influence des produits (alcool, drogue) (Guyon, 2003).

\section{CONCLUSION ET RECOMMANDATIONS}

A partir des études épidémiologiques récentes menées sur les patients des centres de soins spécialisés en toxicomanie, différents sous-groupes 
peuvent être analysés et évalués par rapport au traitement: ainsi les femmes constituent un groupe particulier, et parmi elles, les femmes enceintes, avec les questions spécifiques de l'impact de la méthadone durant la grossesse des héroïnomanes.

Les conduites toxicomaniaques restent minoritaires chez les femmes, mais leur gravité est spécifique, du fait d'une entrée plus rapide dans les usages d'héroïne, d'une polyconsommation plus fréquente, avec des médicaments notamment.

Les femmes dépistées en prison signalent souvent une absence de recours aux soins et des difficultés de suivi spécialisé; elles rencontrent des difficultés de tous ordres et le groupe des mères toxicomanes est encore plus marqué par une précarisation de la santé et du système relationnel.

Les femmes enceintes représentent un groupe particulièrement exposé à des risques multiples, du fait même d'antécédents obstétricaux fréquents et de difficultés de grossesse, révélées par le traitement à la méthadone et le suivi médico-psycho-social.

L'évaluation d'une prescription de méthadone, avec suivi par un centre spécialisé durant la grossesse ne montre pas en moyenne d'amélioration rapide des difficultés psychologiques et de l'intégration sociofamiliale. Une stabilisation de l'état psychologique et relationnel des patientes toutefois est observée, alors que la période de grossesse aurait pu entraîner une aggravation.

Comme pour l'ensemble des patients traités avec la méthadone, la question de la durée du traitement est posée, certains cliniciens constatant une efficacité au bout de trois ans. Pour les femmes enceintes, l'efficacité d'un traitement de substitution devrait ensuite prendre en compte l'évolution de l'enfant et de son développement psycho-moteur et affectif.

Une diminution des usages d'héroïne et d'opiacés est rapidement observée, comme pour l'ensemble des patients traités; il est à noter que parallèlement le dosage de méthadone augmente après le premier mois de traitement, en même temps que d'autres traitements psychotropes associés.

Les données de l'entourage montrent une fragilité des relations, avec beaucoup d'insatisfactions pour les patientes enceintes et des séparations familiales pour un certain nombre. Ces constats incitent à des prises en charge attentives sur le plan de la santé mais aussi sur le plan relationnel.

Les difficultés et résistances face aux soins et prises en charge spécialisés en toxicomanie sont particulièrement lourdes pour les femmes enceintes. Parmi les femmes enceintes toxicomanes vues dans les centres spécialisés, 29\% sont sorties du centre au bout d'un mois, 37\% sont suivies moins de six mois. Le dépistage des toxicomanies est à renforcer dans les services en néonatalogie, pour faciliter le traitement avec méthadone et réciproquement pour les équipes spécialisées en toxicomanie la connaissance des grossesses doit faciliter la prise en charge conjointe nécessaire à un projet stable mère-enfant.

\section{BIBLIOGRAPHIE}

Batey R.G., Weissel K. (1993) A 40 month follow-up of pregnant drug using women treated at Westmead Hospital. Drug and Alcohol Review, 12: 265-270.

Boer K., Smit B.J., Van Huis A.M., Hogerzeil H.V (1994) Substance abuse in pregnancy: do we can? Acta paediatrica Scandinava. Supplément, 404: 65-71.

Chang G., Carroll K.M., Behr H.M., Kosten T.R. (1992) Improving treatment outcome in pregnant opiatedependent women. Journal of substance Abuse Treatment, 9: 327-330.

Depetrillo P, Rice J.M. (1995) Methadone dosing and pregnancy: impact on program compliance. International Journal of the Addictions, 30, (2): 207-217.

Facy F. (1999) Toxicomanes et prescription de méthadone. Paris, EDK. 128p.

Finnegan L.P. (1995) Addiction and pregnancy: maternal and child issues. In: Tagliamonte A., Maremmani I., Drug addiction and related clinical problems. New-York, Springer-Verlag Wien: 137-147.

Fischer G., Jagsch R., Eder H., Gombas W., Etzersdorfer P., Schmidl-Mohl C., Schatten C., Weninger M., Aschauer H.N (1999). Comparison of methadone and slow-release morphine maintenance in pregnant addicts. Addiction, 94 (2): 231-239.

Guyon L. (2003) Les femmes et les substances psychoactives: impact des rôles sociaux. In: Facy F., Guyon L, Les toxicomanies. Paris, EDK: 186-203.

Guyon L, De Koninck M, Morissette P, Chayer L. (2003) Maternité et toxicomanie: la réalité plutôt que la fiction. In: Facy F., Guyon L, Les toxicomanies. Paris, EDK: 263274.

Jarvis M.A.E., Wu-Pong S., Kniseley J.S., Schnoll S.H. (1999) Alterations in methadone metabolism during late pregnancy. Journal of Addictive Diseases, 18, (4): 51-61.

Mc Lean A. (1994) 'The little people therapeutic projet' Ethique et valeurs idéologiques à propos de quatre années de travail aux Etats-Unis de 1987 à 1991. Information Psychiatrique, 70 (2): 163-171.

Offidani C., Chiarotti M., De Giovanni N., Falasconi A.M. (1986) Methadone in pregnancy: clinical, toxicological aspects. Clin Toxicol 24: 295-303.

Picard E. (1995) Méthadone and pregnancy. Archives of public health, 53, (5-6): 183-205.

T3E. (1997) Les traitements de substitution en Europe. Cahiers T3E, (2): 189p.

Wieviorka S. (1994) Grossesse et toxicomanie: une multiplicité des risques. Revue du Praticien, 8, (247): 3741. 